\title{
The Onset of Grade $\geq 3$ Neutropenia Is Associated With Longer Overall Survival in Metastatic Colorectal Cancer Patients Treated With Trifluridine/Tipiracil*
}

\author{
JACOPO GIULIANI and ANDREA BONETTI
}

\author{
Department of Oncology, Mater Salutis Hospital, Legnago, Italy
}

\begin{abstract}
Background/Aim: The aim of this report was to evaluate the onset of grade $\geq 3$ neutropenia during the firstcycle in patients with metastatic colorectal cancer (mCRC) treated with trifluridine/tipiracil and its relationship with clinical parameters of interest, such as overall survival (OS). Patients and Methods: We performed a retrospective analysis of all consecutive patients with $m C R C$, treated with trifluridine/tipiracil in third or later treatment lines in $m C R C$, at our Medical Oncology Unit between July 2017 and December 2018. We evaluated 15 patients. Results: Median age was 63 years (range: 46-80 years). Trifluridineltipiracil was used in third-line treatment in 10 patients (66.7\%). The only serious adverse events were grade $3(26.7 \%)$ and grade $4(13.3 \%)$ neutropenia. Median follow-up time (FUT) was 53 months (range=17-91 months). At the last FUT, 5 patients (33.3\%) were deceased. Median OS was 5 months (range $=1$ 15 months). At the univariate analysis, the onset of grade $\geq 3$ neutropenia at the first cycle showed a statistically positive impact on $O S(p=0.046)$. Conclusion: The onset of grade $\geq 3$ neutropenia at the first-cycle is associated with longer median OS compared to no onset of neutropenia also in real-life.
\end{abstract}

Recently, trifluridine/tipiracil, a new oral combination therapy, offered a therapeutic possibility beyond the secondline for patients affected by metastatic colorectal cancer (mCRC), with improvement in clinical outcomes and prolonged survival (1). In particular, an interesting topic is represented by an association between the onset of

*Presented at the 40th EORTC-PAMM Winter Meeting, February 2019, Verona, Italy.

Correspondence to: Jacopo Giuliani, Department of Oncology, Mater Salutis Hospital, Az. ULSS 9 Scaligera, Via Gianella 1-37045 Legnago (VR), Italy. Tel: +39 0442622364, Fax: +39 0442622469, e-mail: jacopo.giuliani@aulss9.veneto.it

Key Words: Metastatic colorectal cancer, trifluridine/tipiracil, neutropenia. neutropenia and survival in $\mathrm{mCRC}$ treated with trifluridine/ tipiracil. Ohtsu et al., (2) have presented a post-hoc analysis of RECOURSE trial (1) and they have found that the onset of grade $\geq 3$ neutropenia during any cycle was associated with longer median progression free survival (PFS) and overall survival (OS) compared to no onset of neutropenia, similar to those reported by other Authors $(3,4)$.

No real-life data concerning the association between the onset of neutropenia and cancer outcomes in mCRC patients treated with trifluridine/tipiracil have been reported in literature until now (only post-hoc analysis of RCTs presented at International Congress as abstracts) (1, $3,4)$. The aim of this report is to evaluate the possible correlation between the onset of grade $\geq 3$ neutropenia during the first-cycle in patients with mCRC treated with trifluridine/tipiracil and OS.

\section{Patients and Methods}

A retrospective analysis of all consecutive patients with mCRC treated with trifluridine/tipiracil in third or later treatment lines in mCRC at our Medical Oncology Unit between July 2017 and December 2018 was performed. All information was obtained from the patients' clinical records and review of their medical history. Treatment toxicity and antitumoral activity were evaluated according to the National Cancer Institute Common Terminology Criteria for Adverse Events (NCI-CTCAE) version 5.0 (5) and Response Evaluation Criteria In Solid Tumors (RECIST) (6), respectively. Follow-up time (FUT) was defined as the time patients were followed at Our Institution. OS was estimated starting from the first day of the first cycle of trifluridine/tipiracil to the last visit or the patient's death date, censoring surviving patients at the time of last follow-up. A univariate analysis was estimated according to the Kaplan-Meier method with statistical significance $(p<0.05)$ of differences being estimated by the log-rank test. We chose to consider only OS and not PFS because, in the absence of a prospective design to determine whether disease progression occurred at defined intervals, this measurement is fraught with potential bias, as patients may be followed with differing frequencies depending on whether or not they are in a clinical trial, or which therapy they are receiving. The chi-square test, $t$-test, or Fisher's exact test were used to exclude any possible relationship between the results and the different prognostic variables. 


\section{Results}

We evaluated 15 patients with mCRC treated with trifluridine/tipiracil. Four patients $(26.7 \%)$ were female. Median age was 63 years (range $=46-80$ ). KRAS, NRAS and BRAF were wild-type in 7 (46.7\%), 14 (93.3\%) and 15 $(100.0 \%)$ patients, respectively. Trifluridine/tipiracil was used in third-line treatment in 10 patients $(66.7 \%)$ and in fourth-line in the remaining 5 patients $(33.0 \%)$, with a mean of 5 cycles for patient (range=1-14). Eastern Cooperative Oncology Group Performance Status (ECOG PS) before starting trifluridine/tipiracil was 0 in 13 patients $(86.7 \%)$ and 1 in 2 patients (13.3\%), while it was 0 in 10 patients $(66.7 \%), 1$ in 1 patient $(6.7 \%)$ and $\geq 2$ in 4 patients $(26.6 \%)$ after the treatment with trifluridine/tipiracil. One patient $(6.7 \%)$ showed complete remission, one patient (6.7\%) partial remission (PR), 6 patients $(40.0 \%)$ stable disease (SD) and 7 patients (46.7\%) progression of the disease (PD) as their best response to treatment. Grade 3 $(26.7 \%)$ and grade $4(13.3 \%)$ neutropenia were the only serious adverse events reported (in all cases at the firstcycle and confirmed at the subsequent cycles). When grade 3 or 4 neutropenia did not appear at the first-cycle, we did not observe neutropenia in subsequent cycles. Median follow-up time (FUT) was 53 months (range=17-91 months). At the last FUT, 5 patients (33.3\%) were deceased and 10 patients $(66.7 \%)$ were still alive. Median OS was 5 months (range $=1-15$ months). At the univariate analysis, the onset of grade $\geq 3$ neutropenia at the first cycle showed a statisticalyl positive impact on OS $(p=0.046)$ (Figure 1). $\chi^{2}$-test showed a statistically significant relationship between the onset of grade $\geq 3$ neutropenia and the best response to the treatment with trifluridine/tipiracil $(p=0.04)$.

\section{Discussion}

We are aware of the limitations of a retrospective experience, the small size of the cohorts and the fact that data coming from a single Institution could reflect only the habits of that particular set of physicians; however, studies like the above, though the analysis of not selected case studies, are able to evaluate the real-life. In fact, the patients described here represent the complete series and consecutive of patients who underwent a systemic treatment at our Unit in the considered time frame, were treated in a homogeneous way and carefully staged before the beginning of treatment and at regular intervals thereafter. Furthermore, follow up was complete for all patients.

Taken together, our analysis showed that the onset of grade $\geq 3$ neutropenia at the first-cycle is associated with longer median OS compared to no onset of neutropenia in real-life.

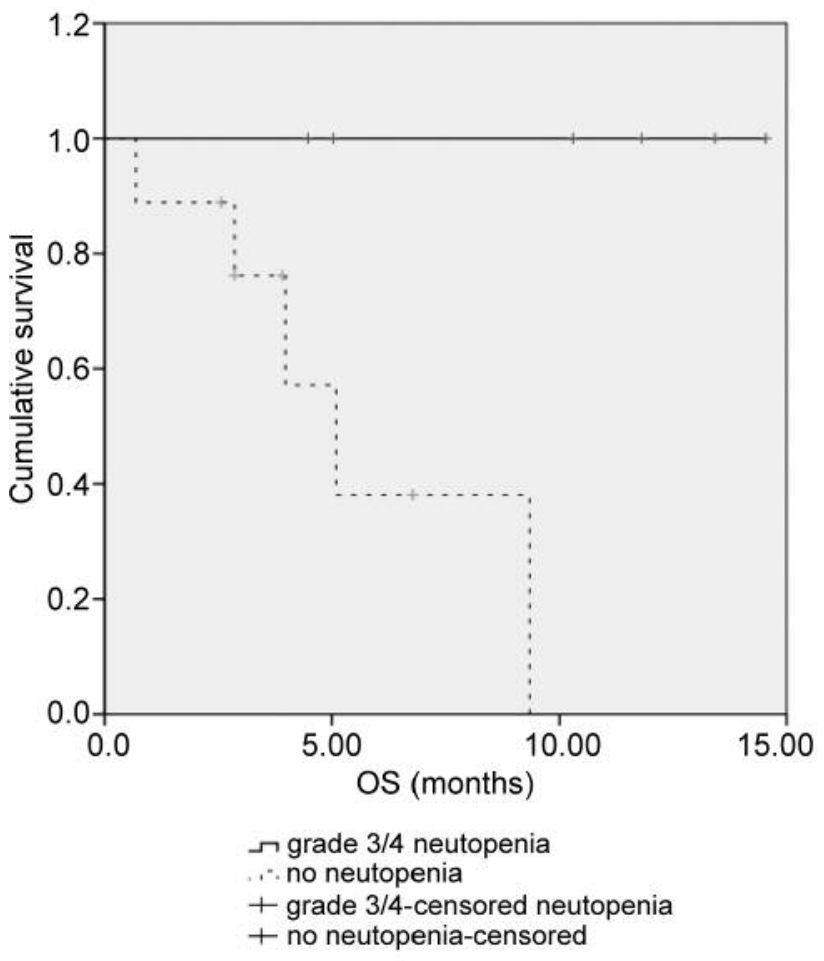

Figure 1. Univariate analysis for overall survival (OS) considering the onset of grade $\geq 3$ neutropenia at the first-cycle in $m C R C$ patients treated with trifluridine/tipiracil in third or subsequent lines.

\section{Conflicts of Interest}

The Authors declare they have no conflicts of interest.

\section{Authors' Contributions}

JG and $\mathrm{AB}$ contributed equally to the conception and design of the study, acquisition, analysis and interpretation of data, drafting the article and revising it critically for important intellectual content, as well as for its final approval to be published.

\section{Acknowledgements}

No funds were received to support this article.

\section{References}

1 Mayer RJ, Van Cutsem E, Falcone A, Yoshino T, GarciaCarbonero R, Mizunuma N, Yamazaki K, Shimada Y, Tabernero J, Komatsu Y, Sobrero A, Boucher E, Peeters M, Tran B, Lenz HJ, Zaniboni A, Hochster H, Cleary JM, Prenen H, Benedetti F, Mizuguchi H, Makris L, Ito M, Ohtsu A; RECOURSE Study Group: Randomized trial of TAS-102 for refractory metastatic colorectal cancer. N Engl J Med 372(20): 1909-1919, 2015. PMID: 25970050. DOI: 10.1056/NEJMoa1414325 
2 Ohtsu A, Yoshino T, Falcone A, Carbonero RG, Argiles G, Sobrero A, Peeters M, Makris L, Benedetti FM, Zaniboni A, Shimada Y, Yamazaki K, Komatsu Y, Hochster HS, Lenz HJ, Tran B, Yoshida K, Van Cutsem E and Mayer RJ: Onset of neutropenia as an indicator of treatment response in the phase 3 RECOURSE trial of TAS-102 vs. placebo in patients with metastatic colorectal cancer. Presented at ASCO 2016 Annual Meeting, Chicago IL, J Clin Oncol 34: abstr.3556, 2016. DOI: 10.1200/JCO.2017.35.4_suppl.775

3 Hamauchi S, Yamazaki K, Masuishi T, Kito Y, Komori A, Tsushima T, Todaka A, Yokota T, Machida N, Fukutomi A, Onozawa Y and Muro K, Yasui H, Mori K and Taniguchi H: Neutropenia as a predictive factor in patients with metastatic colorectal cancer treated with TAS-102. Ann Oncol 26: abstr.165P, 2015. DOI: 10.1093/annonc/mdv523

4 Kasi AM, Kotani D, Cecchini M, Shitara K, Ohtsu A, Ramanathan RK, Hochster HS, Grothey A and Yoshino T: Association of chemotherapy induced neutropenia at 1-month mark (CIN-1-month) and overall survival in patients receiving TAS-102 for refractory metastatic colorectal cancer: a cohort study. J Clin Oncol 34: abstr.e15124, 2016.
5 Common Terminology Criteria for Adverse Events (CTCAE) version 5.0. National Cancer Institute, 2017. https://ctep.cancer. gov/protocolDevelopment/electronic_applications/ctc.htm.

6 Eisenhauer EA, Therasse P, Bogaerts J, Schwartz LH, Sargent D, Ford R, Dancey J, Arbuck S, Gwyther S, Mooney M, Rubinstein L,Shankar L, Dodd L, Kaplan R, Lacombe D and Verweij J: New response evaluation criteria in solid tumours: revised RECIST guideline(version 1.1). Eur J Cancer 45(2): 228247, 2009. PMID: 19097774. DOI: 10.1016/j.ejca.2008.10.026.

Received April 5, 2019

Revised May 25, 2019

Accepted May 28, 2019 